ISSN 1980-5098

\title{
SIMILARIDADE FLORÍSTICA ENTRE TRECHOS DE FLORESTA ESTACIONAL SEMIDECIDUAL DO CORREDOR DE BIODIVERSIDADE SANTA MARIA - PR
}

\author{
FLORISTIC SIMILARITY IN STRETCHES OF A SEASONAL SEMIDECIDUAL FOREST OF \\ SANTA MARIA BIODIVERSITY CORRIDOR, PARANÁ STATE
}

\author{
Darlene Gris ${ }^{1}$ Lívia Godinho Temponi ${ }^{2}$
}

\begin{abstract}
RESUMO
Os Corredores de Biodiversidade têm a função de manter e restabelecer a conectividade entre fragmentos florestais remanescentes. O Corredor de Biodiversidade Santa Maria - PR visa conectar o Parque Nacional do Iguaçu à mata ciliar do reservatório de Itaipu, através da implantação de áreas de reflorestamento entre fragmentos remanescentes de Floresta Estacional Semidecidual. Este trabalho objetivou avaliar a diversidade de espécies arbóreas e a similaridade florística entre áreas de florestas nativas secundárias e de reflorestamentos em diferentes idades. Compararam-se a densidade, a diversidade, a equabilidade e a composição florística dos cinco fragmentos e a similaridade foi avaliada pelos índices de Jaccard e de distância de Bray-Curtis. Em dois hectares amostrados, foram encontrados 2328 indivíduos, distribuídos em 134 espécies e 39 famílias. A similaridade florística agrupou as florestas secundárias nativas mais preservadas e as áreas com reflorestamento em grupos distintos. As 11 espécies exóticas encontradas nas áreas de reflorestamento não foram observadas nas áreas mais preservadas e, portanto, o manejo das mesmas deve ser incentivado, como medida de preservação dos fragmentos nativos remanescentes de Floresta Estacional Semidecidual.
\end{abstract}

Palavras-chave: Mata Atlântica; fragmentação.

\begin{abstract}
The function of Biodiversity Corridors is to maintain and restore connectivity between forest fragments remains. Santa Maria Biodiversity Corridor, in PR state, aims to connect Iguaçu National Park to the riparian forest of the Itaipu reservoir, through the implementation of reforestation areas between remnants of Seasonal Semidecidual Forest. This study aimed to evaluate the diversity of tree species and the floristic similarity between the areas of native secondary forests and reforested areas of different ages. There were compared the density values, diversity, evenness, and floristic composition from five study areas, and the similarity was evaluated by Jaccard and Bray-Curtis distance. In two hectares, we found 2,328 individuals of 134 species and 39 families. The floristic similarity grouped the native secondary forests, which are best preserved, and the reforested areas in different groups. The 11 exotic species found in reforested areas were not observed in the most preserved areas. So, the managament of them should be encouraged in order to protect the remaining Seasonal Semidecidual Forest.
\end{abstract}

Keywords: Atlantic Rainforest; fragmentation.

1 Bióloga, MSc., Doutoranda em Ecologia e Conservação, Centro de Ciências Biológicas e da Saúde, Universidade Federal de Mato Grosso do Sul, Cidade Universitária, s/n, Universitário, CEP 79070-900, Campo Grande (MS), Brasil. darlenegris@hotmail.com

2 Bióloga, Dra., Professora Adjunta, Curadora do Herbário UNOP, Centro de Ciências Biológicas e da Saúde, Universidade Estadual do Oeste do Paraná, Rua Universitária, 2069, Jardim Universitário, CEP 85819-110, Cascavel (PR), Brasil. liviatemponi@yahoo.com.br

Recebido para publicação em 20/10/2014 e aceito em 24/11/2015

Ci. Fl., v. 27, n. 3, jul.-set., 2017 


\section{INTRODUÇÃO}

A Mata Atlântica é uma das florestas mais ameaçadas do planeta, sendo considerada um dos hotspots de conservação mundiais (MYERS et al., 2000). Essa formação vem sofrendo modificações e destruições severas, especialmente pela perda e fragmentação do ecossistema, formando florestas em manchas isoladas circundadas por áreas transformadas pela ação antrópica. A fragmentação pode aumentar o efeito de borda e o isolamento do ecossistema, dificultando a sobrevivência de espécies, especialmente as endêmicas e as ameaçadas de extinção (CAMPOS, 2006; PINTO et al., 2006).

As regiões que concentram as maiores áreas de remanescentes florestais estão associadas a unidades de conservação de proteção integral, porém, esses remanescentes encontram-se isolados uns dos outros. Dessa forma, a conservação da biodiversidade depende da expansão da rede de áreas protegidas, tanto públicas quanto privadas, e de áreas que não são protegidas por lei (PINTO et al., 2006). Mais que isso, sua conservação depende da conexão entre essas áreas florestais próximas, permitindo a sobrevivência das metapopulações, aumentando a variabilidade genética pelo fluxo gênico e protegendo a biodiversidade desses remanescentes florestais (HANSKI; GILPIN, 1991).

Neste contexto surgem os Corredores de Biodiversidade, porções de ecossistemas naturais ou seminaturais, que têm a função de manter e/ou restabelecer a conectividade em uma paisagem (CAMPOS, 2006; ROCHA et al., 2006), desde que seja atingido o limiar de conectividade estrutural (METZGER; DÉCAMPS, 1997).

No Brasil, existem várias iniciativas de corredores de biodiversidade. Um destes corredores abrange as bacias dos rios Paraná e Iguaçu, compreendendo a Bacia do Rio Paraná III, os Parques Nacionais da Ilha Grande e do Iguaçu (PR), o Parque Estadual do Turvo (RS), a Área de Proteção Ambiental Federal das Ilhas e Várzeas do Rio Paraná e o Parque Estadual do Morro do Diabo (SP) (ITAIPU BINACIONAL, 2010). Parte do referido corredor é o chamado Corredor de Biodiversidade Santa Maria, estabelecido em 2003, visando conectar o Parque Nacional do Iguaçu à mata ciliar da borda do lago de Itaipu (ITAIPU BINACIONAL, 2010; IBAMA, 2012).

As áreas do Corredor de Biodiversidade Santa Maria (CBSM) encontram-se em distintos estágios de regeneração e consequentemente, composições florísticas e estruturas da vegetação. No entanto, ainda não foram descritos estudos deste caráter no CBSM.

Assim, o objetivo desse trabalho foi avaliar a composição florística, diversidade e similaridade florística entre áreas de florestas nativas secundárias e de reflorestamentos em diferentes idades na Floresta Estacional Semidecidual (FES) do Corredor de Biodiversidade Santa Maria, oeste do Paraná,

\section{MATERIAL E MÉTODOS}

\section{Área de estudo}

O Corredor de Biodiversidade Santa Maria (CBSM) situa-se entre o Parque Nacional do Iguaçu e o Fragmento Reflorestado Tardio, distantes cerca de $15 \mathrm{~km}$ um do outro. Nessa extensão foram implantadas 50 parcelas de $20 \times 20 \mathrm{~m}$ distantes $20 \mathrm{~m}$ umas das outras. Foram implantadas dez parcelas, em cada uma das cinco áreas: Parque Nacional do Iguaçu (PNI); Área de Preservação Permanente do Lago de Itaipu (Fragmento Reflorestado Tardio (FRt)); RPPN Fazenda Santa Maria (Fragmento Tardio (FT)); Fragmento Secundário (FS); e Fragmento Reflorestado Inicial (FRi) (Figura 1).

O Parque Nacional do Iguaçu (PNI) está situado no extremo sul do CBSM, possuindo área total de 185.262 hectares (SALAMUNI et al., 2002). A área de amostragem corresponde a uma das mais estreitas do PNI, próxima ao Rio Iguaçu, mas não sujeita ao alagamento. A vegetação encontra-se em estágio avançado de sucessão/regeneração, porém, essa área ainda é utilizada, eventualmente, para extração de palmito e como acesso ao rio para pesca clandestina e caça. A área estudada situa-se entre as coordenadas

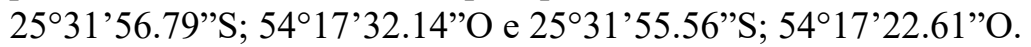

No extremo norte do CBSM, encontra-se a Área de Preservação Permanente do Lago de Itaipu (FRt) com aproximadamente $2900 \mathrm{~km}$ de extensão e uma largura média de 217 metros. Esse reflorestamento iniciou-se em 1979 como parte do processo de compensação da Itaipu Binacional pela construção do 


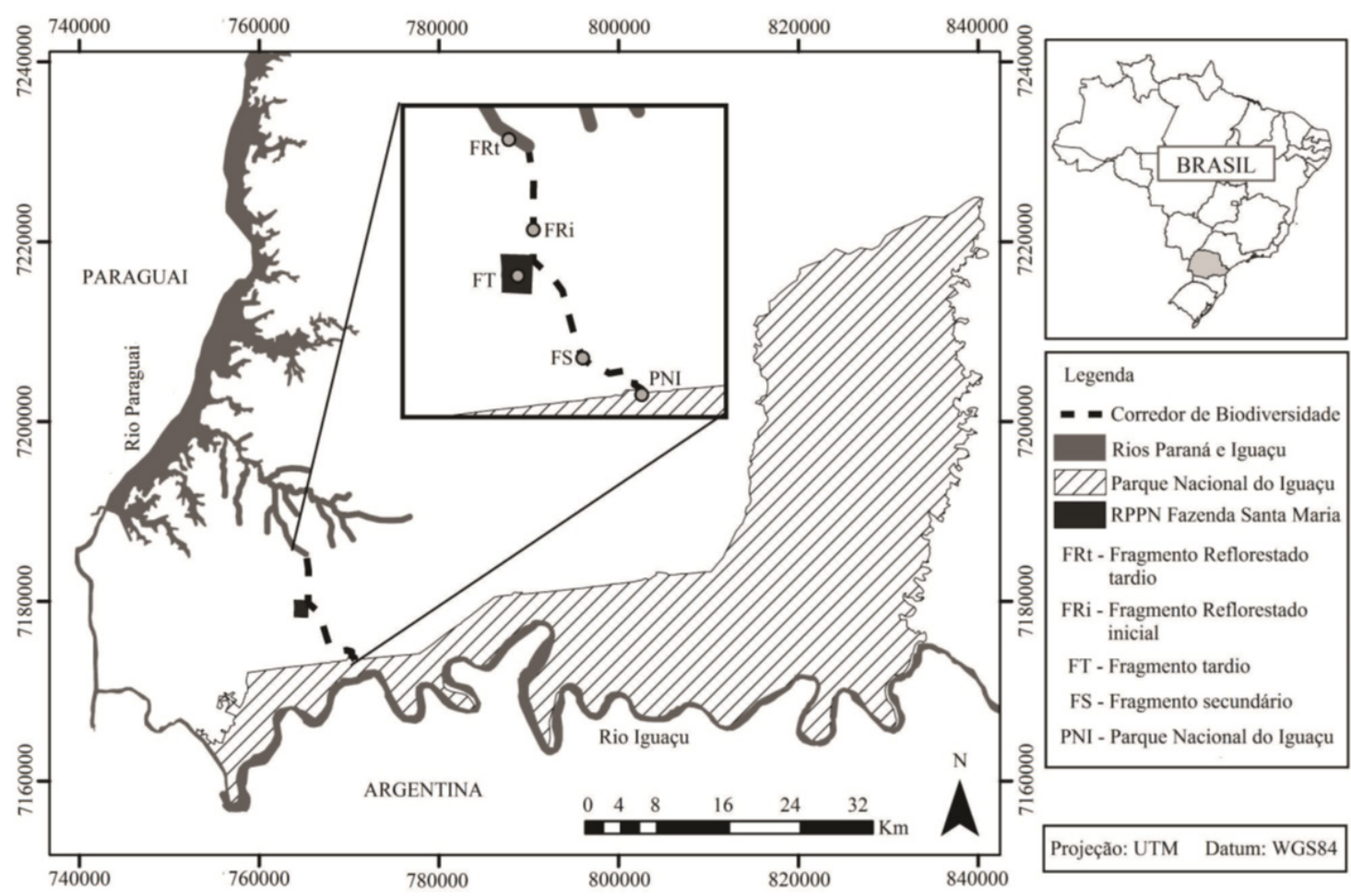

FIGURA 1: Localização do Corredor de Biodiversidade Santa Maria, Paraná, Brasil (CBSM). Distribuição das cinco áreas de estudo ao longo do CBSM.

FIGURE 1: Location of the five study areas along Santa Maria Biodiversity Corridor (CBSM) in west Paraná state, Brazil.

lago (IBAMA, 2012). O plantio incluiu espécies nativas e exóticas, principalmente frutíferas, e também a manutenção da vegetação já existente nessa área, esse reflorestamento apresenta áreas com predominância de Mangifera indica L. e de Psidium guajava L. sendo uma área sujeita a impactos casuais, como pisoteio, relacionados às atividades agropecuárias das fazendas do entorno. A área amostrada encontra-se entre as coordenadas $25^{\circ} 24^{\prime} 3.72^{\prime \prime} \mathrm{S} ; 54^{\circ} 23^{\prime} 41.12^{\prime \prime O}$ e $25^{\circ} 24^{\prime} 14.60^{\prime \prime} \mathrm{S} ; 4^{\circ} 23^{\prime} 41.96^{\prime \prime} \mathrm{O}$.

O maior fragmento entre estes dois extremos é a RPPN Fazenda Santa Maria (FT), com aproximadamente 242 ha. A área amostrada situa-se entre as coordenadas $25^{\circ} 29^{\prime} 30.70^{\prime \prime} \mathrm{S} ; 54^{\circ} 21^{\prime} 30.61^{\prime \prime} \mathrm{O}$

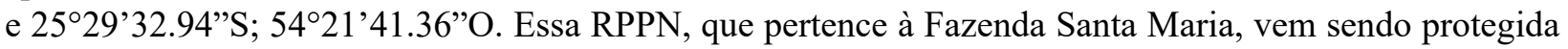
desde o ano de 1955 e tornou-se Unidade de Conservação no ano de 1997. Essa área, atravessada por pequenos riachos, possui um histórico de ações como caça e extração de madeira anterior a sua proteção, contudo, hoje, a vegetação encontra-se em estágio avançado de regeneração já que a mesma não sofre nenhum tipo de ação antrópica há aproximadamente 60 anos.

Entre o PNI e a FT foi estabelecida uma área de estudo, localizada entre as coordenadas

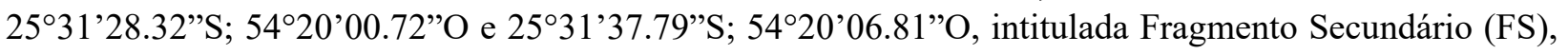
de aproximadamente 87 ha, pertencente à Fazenda São José, a menos de $700 \mathrm{~m}$ da borda do PNI. Esta área possui um histórico de atividades antrópicas, como caça e extração de madeira e palmito e, devido a essas atividades, clareiras são bastante comuns, bem como intenso efeito de borda por ser uma área pequena e protegida a pouco tempo. Porém, apesar de não possuir título de Unidade de Conservação, vem sendo preservada, há algumas décadas, como remanescente florestal da fazenda, sendo excluídas desde então, qualquer atividade antrópica, dando espaço à regeneração natural do remanescente.

Também foi estudado um Fragmento Reflorestado Inicial (FRi), que se situa entre o FT e o FRt, entre as coordenadas $25^{\circ} 29^{\prime} 00.84^{\prime \prime} \mathrm{S} ; 4^{\circ} 21^{\prime} 16.53^{\prime \prime O}$ e $25^{\circ} 28^{\prime} 50,50^{\prime \prime} \mathrm{S} 54^{\circ} 21^{\prime} 16,80^{\prime \prime} \mathrm{O}$. Esta área, reflorestada 
pela Itaipu Binacional em parceria com a Fazenda Santa Maria a partir de maio de 2003, corresponde a uma faixa de $60 \mathrm{~m}$ de largura com, aproximadamente, $30 \mathrm{ha}$ em toda a sua extensão. O plantio nessa faixa de reflorestamento foi realizado em linhas, com uma baixa riqueza de espécies, a maioria frutíferas, tanto nativas quanto exóticas, bem como muitas espécies da família Fabaceae.

Todas as áreas amostradas pertencem à unidade morfoescultural Terceiro Planalto Paranaense, no Planalto de Foz do Iguaçu, com altitudes que variam de 120 a 540 m (SANTOS et al., 2006; MAACK, 2012). O clima local é subtropical úmido (Cfa) e o solo do tipo latossolo vermelho. A temperatura média anual para a região é de $21,55^{\circ} \mathrm{C}$ e a pluviometria anual média em torno $1803 \mathrm{~mm}$, sendo que dois terços das chuvas estão distribuídas entre os meses de outubro a março, principalmente dezembro e janeiro (BHERING; SANTOS, 2008; SISTEMA METEOROLÓGICO DO PARANÁ, 2011). A vegetação é predominantemente formada por Floresta Estacional Semidecidual Submontana (VELOSO; FILHO; LIMA, 1991) com áreas nativas e áreas de reflorestamento em diferentes graus de preservação.

\section{Coleta de dados e análises}

Foram incluídos no levantamento os indivíduos de porte arbóreo cuja circunferência à altura do peito (CAP) foi igual ou superior a $15 \mathrm{~cm}$ e, para indivíduos com mais de um caule adotou-se que pelo menos um deles deveria possuir diâmetro igual ou superior a $15 \mathrm{~cm}$ (RODRIGUES; GANDOLFI, 2004). Os indivíduos amostrados foram numerados e visitados mensalmente (setembro de 2010 a novembro de 2011) com o intuito de coletar todos os indivíduos em seu período de floração. As amostras foram preparadas de acordo com técnicas usuais de herborização (BRIDSON; FORMAN, 2004), sendo as exsicatas inseridas no Herbário da Universidade Estadual do Oeste do Paraná (UNOP).

Para identificação do material botânico foram utilizadas listagens florísticas regionais, chaves de identificação, bibliografia específica (LORENZI, 2002a; 2002b; 2010; RAMOS et al., 2008) e consultas a especialistas e a exsicatas nos herbários UNOP e Herbário do Museu Botânico Municipal de Curitiba (MBM).

As listagens foram produzidas com as famílias botânicas organizadas de acordo com o sistema de classificação atual (ANGIOSPERM PHYLOGENY GROUP, 2009) e os nomes dos autores das espécies de acordo com Lista de Espécies da Flora do Brasil (JARDIM BOTÂNICO DO RIO DE JANEIRO, 2015).

As espécies mais abundantes em cada uma das áreas foram classificadas de acordo com sua síndrome de dispersão (VANDER PIJL, 1982) em zoocóricas, anemóricas e autocóricas, para tanto também foi utilizado como base o trabalho desenvolvido por Toscan et al. (2014) em áreas do mesmo corredor.

A densidade, o índice de diversidade de Shannon-Wiener $\left(\mathrm{H}^{\prime}\right)$ e o índice de equabilidade de Pielou (J') foram calculadas com o auxílio do Programa FITOPAC 2.1.2 (SHEPHERD, 2011). Para comparar o índice de diversidade utilizou-se o teste $t$ de Hutcheson ao nível de $5 \%(\mathrm{p}<0,05)$ e, para tanto, as diversidades das áreas foram comparadas par a par (HUTCHESON, 1970).

Foram elaboradas matrizes, usando os dados de presença/ausência e as densidades das espécies encontradas em cada parcela estudada. Estas matrizes foram padronizadas com as diferentes informações e utilizadas para a análise de similaridade de Jaccard (MUELLER-DOMBOIS; ELLENBERG, 1974) e dissimilaridade utilizando o índice de distância de Bray-Curtis (POLA, 2010) e as análises de agrupamento das localidades pelo método de média de grupo (UPGMA), obtidos através do software R 3.0.1 (R CORE TEAM, 2013).

\section{RESULTADOS E DISCUSSÃO}

\section{Diversidade e levantamento florístico}

Nas cinco áreas estudadas foram amostrados 2328 indivíduos, pertencentes a 134 espécies e 39 famílias botânicas (Tabela 1).

Apenas quatro espécies ocorreram em todas as áreas amostradas, sendo elas: Syagrus romanzoffiana; Inga marginata; Peltophorum dubium e Cecropia pachystachya. Enquanto as espécies: Annona sylvatica; Tabernaemontana hystrix; Jacaranda micrantha; Alchornea triplinervia; Lonchocarpus cultratus; 
TABELA 1: Famílias, espécies e número de indivíduos para as cinco áreas de estudo no Corredor de Biodiversidade Santa Maria (PR). Parque Nacional do Iguaçu (PNI), Fragmento Secundário (FS), Fragmento Tardio (FT), Fragmento Reflorestado Inicial (FRi) e Fragmento Reflorestado Tardio (FRt).

TABLE 1: Families, species, and number of individuals for the five sampled areas in Santa Maria Biodiversity Corridor, PR state. Iguaçu National Park (PNI), Secondary Forest Fragment (FS), Old-Forest Fragment (FT), Initial Reforested Fragment (FRi) and Late Reforested Fragment (FRt).

\begin{tabular}{|c|c|c|c|c|c|c|}
\hline Família & Nome científico & PNI & FS & FT & FRi & FRt \\
\hline \multirow[t]{2}{*}{ Anarcadiaceae } & Astronium graveolens Jacq. & 0 & 0 & 3 & 4 & 0 \\
\hline & Mangifera indica $\mathrm{L} . *$ & 0 & 0 & 0 & 0 & 10 \\
\hline \multirow[t]{3}{*}{ Annonaceae } & Annona neosalicifolia H.Rainer & 0 & 0 & 1 & 0 & 0 \\
\hline & Annona neosericea H.Rainer & 0 & 0 & 0 & 0 & 1 \\
\hline & Annona sylvatica A.St.-Hil. & 1 & 1 & 4 & 0 & 3 \\
\hline \multirow[t]{4}{*}{ Apocynaceae } & Aspidosperma polyneuron Müll.Arg. & 5 & 0 & 13 & 0 & 0 \\
\hline & Aspidosperma tomentosum Mart. & 0 & 0 & 1 & 0 & 0 \\
\hline & Rauvolfia sellowii Müll. Arg. & 3 & 0 & 0 & 0 & 0 \\
\hline & Tabernaemontana hystrix Steud & 0 & 2 & 1 & 9 & 3 \\
\hline \multirow[t]{2}{*}{ Araliaceae } & Dendropanax cuneatus (DC.) Decne. \& Planch. & 0 & 0 & 9 & 0 & 0 \\
\hline & Schefflera calva (Cham.) Frodin \& Fiaschi & 0 & 0 & 3 & 0 & 0 \\
\hline \multirow[t]{2}{*}{ Arecaceae } & Euterpe edulis Mart & 159 & 0 & 125 & 0 & 0 \\
\hline & Syagrus romanzoffiana (Cham.) Glassman & 5 & 4 & 17 & 3 & 1 \\
\hline \multirow[t]{3}{*}{ Bignoniaceae } & Handroanthus impetiginosus Mattos & 0 & 0 & 0 & 55 & 0 \\
\hline & Handroanthus ochraceus (Cham.) Mattos & 0 & 0 & 0 & 0 & 2 \\
\hline & Jacaranda micrantha Cham. & 1 & 1 & 3 & 0 & 11 \\
\hline \multirow[t]{5}{*}{ Boraginaceae } & Cordia americana (L.) Gottschling \&J.S.Mill. & 0 & 0 & 1 & 0 & 11 \\
\hline & Cordia ecalyculata Vell. & 2 & 4 & 13 & 0 & 0 \\
\hline & Cordia rufescens A.DC. & 0 & 1 & 0 & 0 & 0 \\
\hline & Cordia superba Cham. & 0 & 1 & 0 & 0 & 0 \\
\hline & Cordia trichotoma (Vell.) Arráb. ex Steud. & 0 & 3 & 0 & 0 & 1 \\
\hline \multirow[t]{2}{*}{ Cannabaceae } & Celtis fluminensis Carauta & 0 & 2 & 0 & 0 & 1 \\
\hline & Trema micrantha $(\mathrm{L}$.$) Blume$ & 1 & 0 & 1 & 2 & 0 \\
\hline Cardiopteridaceae & Citronella paniculata (Mart.) R.A.Howard & 2 & 1 & 5 & 0 & 0 \\
\hline Caricaceae & Jacaratia spinosa (Aubl.) A.DC. & 0 & 0 & 9 & 0 & 0 \\
\hline Casuarinaceae & Casuarina equisetifolia $\mathrm{L} . *$ & 0 & 0 & 0 & 10 & 0 \\
\hline Celastraceae & Peritassa campestris (Cambess.) A.C.Sm. & 0 & 0 & 1 & 0 & 0 \\
\hline Elaeocarpaceae & Sloanea hirsuta (Schott) Planch. ex Benth. & 0 & 1 & 0 & 0 & 0 \\
\hline \multirow[t]{6}{*}{ Euphorbiaceae } & Actinostemon concolor (Spreng.) Müll.Arg. & 4 & 2 & 2 & 0 & 0 \\
\hline & Alchornea triplinervia (Spreng.) Müll.Arg. & 12 & 5 & 11 & 3 & 0 \\
\hline & Croton floribundus Spreng. & 0 & 0 & 0 & 1 & 0 \\
\hline & Manihot grahamii Hook. & 0 & 0 & 0 & 2 & 0 \\
\hline & Sebastiania brasiliensis Spreng. & 0 & 15 & 0 & 0 & 0 \\
\hline & Sebastiania commersoniana (Baill.) L.B.Sm. \& Downs & 0 & 0 & 0 & 0 & 3 \\
\hline \multirow[t]{7}{*}{ Fabaceae } & Anadenanthera peregrina (L.) Speg. & 0 & 0 & 0 & 0 & 10 \\
\hline & Apuleia leiocarpa (Vogel) J.F.Macbr. & 1 & 0 & 0 & 0 & 0 \\
\hline & Bauhinia forficata Link & 0 & 0 & 0 & 0 & 1 \\
\hline & Calliandra foliolosa Benth. & 1 & 14 & 1 & 0 & 0 \\
\hline & Cassia leptophylla Vogel & 0 & 0 & 0 & 1 & 0 \\
\hline & Copaifera langsdorffii Desf. & 0 & 0 & 0 & 0 & 1 \\
\hline & Dalbergia frutescens (Vell.) Britton & 0 & 1 & 0 & 0 & 0 \\
\hline Fabaceae & Enterolobium contortisiliquum (Vell.) Morong & 1 & 0 & 2 & 0 & 2 \\
\hline
\end{tabular}


TABELA 1: Continuação...

TABLE 1: Continued...

\begin{tabular}{|c|c|c|c|c|c|c|}
\hline Família & Nome científico & PNI & FS & FT & FRi & FRt \\
\hline & Erythrina falcata Benth. & 0 & 1 & 0 & 0 & 0 \\
\hline & Holocalyx balansae Micheli & 2 & 2 & 7 & 0 & 0 \\
\hline & Inga marginata Willd. & 7 & 5 & 3 & 11 & 1 \\
\hline & Inga striata Benth. & 1 & 0 & 1 & 68 & 0 \\
\hline & Leucaena leucocephala (Lam.) de Wit* & 0 & 0 & 0 & 0 & 9 \\
\hline & Lonchocarpus cultratus (Vell.) A.M.G.Azevedo \& H.C.Lima & 1 & 3 & 11 & 17 & 0 \\
\hline & Lonchocarpus muehlbergianus Hassl. & 0 & 0 & 8 & 0 & 0 \\
\hline & Machaerium paraguariense Hassl. & 0 & 1 & 2 & 1 & 5 \\
\hline & Machaerium stipitatum Vogel & 2 & 37 & 4 & 0 & 61 \\
\hline & Mimosa bimucronata (DC.) Kuntze & 0 & 0 & 2 & 5 & 0 \\
\hline & Myroxylon peruiferum L.f. & 0 & 1 & 0 & 0 & 0 \\
\hline & Parapiptadenia rigida (Benth.) Brenan & 6 & 4 & 2 & 0 & 32 \\
\hline & Peltophorum dubium (Spreng.) Taub. & 1 & 4 & 2 & 13 & 5 \\
\hline & Piptadenia gonoacantha (Mart.) J.F.Macbr. & 0 & 0 & 0 & 26 & 0 \\
\hline & Poincianella pluviosa (DC.) L.P.Queiroz & 0 & 0 & 0 & 6 & 0 \\
\hline & Pterogyne nitens Tul. & 0 & 0 & 0 & 7 & 2 \\
\hline & Schizolobium parahyba (Vell.) Blake & 0 & 0 & 0 & 3 & 0 \\
\hline & Senegalia polyphylla (DC.) Britton \& Rose & 0 & 0 & 3 & 43 & 2 \\
\hline & Senegalia recurva (Benth.) Seigler\&Ebinger & 0 & 0 & 0 & 9 & 0 \\
\hline Lamiaceae & Aegiphila integrifolia (Jacq.) Moldenke & 2 & 0 & 4 & 0 & 0 \\
\hline \multirow[t]{6}{*}{ Lauraceae } & Endlicheria paniculata (Spreng.) J.F.Macbr. & 2 & 1 & 9 & 0 & 24 \\
\hline & Nectandra megapotamica (Spreng.) Mez. & 8 & 20 & 9 & 0 & 1 \\
\hline & Ocotea diospyrifolia (Meisn.) Mez & 3 & 12 & 6 & 0 & 5 \\
\hline & Ocotea silvestris Vattimo-Gil & 7 & 2 & 1 & 0 & 2 \\
\hline & Ocotea puberula (Rich.) Nees & 0 & 0 & 1 & 0 & 0 \\
\hline & Persea americana Mill.* & 0 & 0 & 0 & 0 & 1 \\
\hline Loganiaceae & Strychnos trinervis (Vell.) Mart & 1 & 1 & 2 & 0 & 0 \\
\hline \multirow[t]{4}{*}{ Malvaceae } & Ceiba speciosa (A. St.-Hil.) Ravena & 4 & 7 & 5 & 0 & 0 \\
\hline & Guazuma ulmifolia Lam. & 0 & 0 & 0 & 33 & 0 \\
\hline & Heliocarpus americanus $\mathrm{L}$. & 2 & 0 & 0 & 0 & 0 \\
\hline & Luehea divaricata Mart. \&Zucc. & 0 & 5 & 0 & 0 & 19 \\
\hline Melastomataceae & Miconia pusilliflora (DC.) Naudin & 0 & 0 & 1 & 0 & 0 \\
\hline \multirow[t]{9}{*}{ Meliaceae } & Cabralea canjerana (Vell.) Mart. & 7 & 8 & 12 & 0 & 0 \\
\hline & Cedrela fissilis Vell. & 2 & 4 & 12 & 0 & 2 \\
\hline & Guarea guidonia (L.) Sleumer & 1 & 0 & 0 & 0 & 0 \\
\hline & Guarea kunthiana A.Juss. & 57 & 3 & 27 & 0 & 0 \\
\hline & Guarea macrophylla Vahl & 0 & 0 & 1 & 0 & 0 \\
\hline & Trichilia casaretti C.DC. & 0 & 1 & 1 & 0 & 0 \\
\hline & Trichilia catigua A.Juss. & 13 & 9 & 4 & 0 & 0 \\
\hline & Trichilia elegans A.Juss. & 2 & 0 & 0 & 0 & 0 \\
\hline & Trichilia pallida $\mathrm{Sw}$. & 1 & 0 & 0 & 0 & 0 \\
\hline Monimiaceae & Hennecartia omphalandra J.Poiss. & 0 & 8 & 0 & 0 & 0 \\
\hline \multirow[t]{3}{*}{ Moraceae } & Ficus insipida Willd. & 3 & 0 & 0 & 12 & 0 \\
\hline & Maclura tinctoria (L.) D.Don ex Steud. & 9 & 0 & 3 & 0 & 11 \\
\hline & Sorocea bonplandii (Baill.) W.C.Burger et al. & 99 & 23 & 30 & 0 & 0 \\
\hline Myrsinaceae & Myrsine umbellata Mart. & 0 & 3 & 3 & 0 & 0 \\
\hline \multirow[t]{3}{*}{ Myrtaceae } & Calyptranthes concinna DC. & 0 & 0 & 0 & 0 & 2 \\
\hline & Campomanesia guazumifolia (Cambess.) O.Berg & 1 & 3 & 0 & 0 & 0 \\
\hline & Campomanesia xanthocarpa (Mart.) O.Berg & 1 & 17 & 8 & 0 & 0 \\
\hline
\end{tabular}

Continua... 
TABELA 1: Continuação...

TABLE 1: Continued...

\begin{tabular}{|c|c|c|c|c|c|c|}
\hline Família & Nome científico & PNI & FS & FT & FRi & FRt \\
\hline & Eucalyptus polyanthemos Schauer* & 0 & 0 & 0 & 0 & 3 \\
\hline & Eugenia burkartiana (D.Legrand) D.Legrand & 2 & 0 & 2 & 0 & 0 \\
\hline & Eugenia florida DC. & 0 & 0 & 1 & 0 & 0 \\
\hline & Eugenia gracílima Kiaersk. & 0 & 0 & 0 & 0 & 3 \\
\hline & Eugenia hiemalis Cambess. & 0 & 0 & 0 & 0 & 1 \\
\hline & Eugenia involucrata DC. & 0 & 1 & 0 & 0 & 1 \\
\hline & Eugenia pyriformis Cambess. & 0 & 4 & 0 & 0 & 5 \\
\hline & Eugenia ramboi D. Legrand. & 3 & 2 & 4 & 0 & 0 \\
\hline & Myrcia laruotteana Cambess. & 0 & 0 & 0 & 0 & 1 \\
\hline & Plinia rivularis (Cambess.) Rotman & 9 & 1 & 2 & 0 & 0 \\
\hline & Psidium guajava L.* & 0 & 0 & 0 & 0 & 129 \\
\hline Nyctaginaceae & Neea pendulina Heimerl & 0 & 1 & 0 & 0 & 0 \\
\hline Oleaceae & Ligustrum japonicum Thunb.* & 0 & 0 & 0 & 0 & 2 \\
\hline Peraceae & Pera glabrata (Schott) Poepp. ex Baill. & 0 & 0 & 1 & 0 & 0 \\
\hline Phytolaccaceae & Seguieria aculeata Jacq. & 1 & 5 & 1 & 0 & 0 \\
\hline Piperaceae & Piper amalago $\mathrm{L}$. & 2 & 0 & 0 & 0 & 0 \\
\hline Rhamnaceae & HoveniadulcisThunb.* & 0 & 0 & 0 & 0 & 57 \\
\hline \multirow[t]{2}{*}{ Rosaceae } & Eriobotrya japonica (Thunb.) Lindl.* & 0 & 0 & 0 & 0 & 1 \\
\hline & Prunus myrtifolia (L.) Urb. & 0 & 5 & 4 & 0 & 15 \\
\hline \multirow[t]{10}{*}{ Rutaceae } & Balfourodendron riedelianum (Engl.) Engl. & 5 & 11 & 21 & 0 & 0 \\
\hline & Citrus X aurantium L.* & 22 & 9 & 0 & 0 & 4 \\
\hline & Citrus deliciosa Ten.* & 0 & 0 & 0 & 0 & 2 \\
\hline & Citrus limonia Osbeck* & 0 & 0 & 0 & 0 & 4 \\
\hline & Helietta apiculata Benth. & 0 & 0 & 0 & 0 & 20 \\
\hline & Pilocarpus pauciflorus A.St.-Hil. & 1 & 0 & 0 & 0 & 0 \\
\hline & Pilocarpus pennatifolius Lem. & 3 & 1 & 0 & 0 & 0 \\
\hline & Zanthoxylum caribaeum Lam. & 3 & 0 & 0 & 0 & 0 \\
\hline & Zanthoxylum petiolare A.St.-Hil. \& Tul. & 0 & 4 & 2 & 0 & 0 \\
\hline & Zanthoxylum rhoifolium Lam. & 0 & 0 & 0 & 0 & 5 \\
\hline \multirow[t]{3}{*}{ Salicaceae } & Casearia decandra Jacq. & 1 & 4 & 0 & 0 & 0 \\
\hline & Casearia gossypiosperma Briq. & 0 & 0 & 0 & 9 & 0 \\
\hline & Casearia sylvestris Sw. & 2 & 2 & 3 & 0 & 54 \\
\hline \multirow[t]{4}{*}{ Sapindaceae } & Allophylus edulis (A.St.-Hil. et al.) Hieron. ex Niederl. & 1 & 7 & 4 & 0 & 6 \\
\hline & Cupania vernalis Cambess. & 0 & 7 & 0 & 0 & 1 \\
\hline & Diatenopteryx sorbifolia Radlk. & 4 & 13 & 0 & 0 & 5 \\
\hline & Matayba elaeagnoides Radlk. & 0 & 1 & 1 & 0 & 3 \\
\hline \multirow[t]{2}{*}{ Sapotaceae } & Chrysophyllum gonocarpum (Mart. \& Eichler ex Miq.) Engl. & 9 & 26 & 34 & 0 & 0 \\
\hline & Chrysophyllum marginatum (Hook. \& Arn.) Radlk. & 4 & 8 & 8 & 0 & 0 \\
\hline \multirow[t]{2}{*}{ Solanaceae } & Cestrum bracteatum Link \& Otto & 0 & 0 & 1 & 0 & 0 \\
\hline & Solanum argenteum Dunal & 0 & 1 & 1 & 0 & 0 \\
\hline \multirow[t]{2}{*}{ Urticaceae } & Cecropia pachystachya Trécul & 3 & 7 & 7 & 1 & 9 \\
\hline & Urera baccifera (L.) Gaudich. ex Wedd. & 0 & 1 & 6 & 0 & 0 \\
\hline Violaceae & Pombalia bigibbosa (A.St.Hil.) Paula-Souza & 0 & 1 & 1 & 0 & 0 \\
\hline Total & & 518 & 365 & 514 & 355 & 576 \\
\hline
\end{tabular}

Em que: * Espécies exóticas no Brasil 
Machaerium paraguariense; Machaerium stipitatum; Parapiptadenia rigida; Endlicheria paniculata; Nectandra megapotamica; Ocotea diospyrifolia; Ocotea silvestris; Cedrela fissilis; Casearia sylvestris e Allophylus edulis ocorreram em quatro das cinco áreas estudadas. Essas espécies são nativas e comuns em áreas de FES, sendo amplamente registradas por diversos autores em trabalhos em mesma formação vegetacional (BIANCHINI et al., 2003; CAMPOS; ROMAGNOLO; SOUZA, 2000; COSTA-FILHO; NANNI; CAMPOS, 2006; DEL QUIQUI et al., 2007; IVANAUSKAS; RODRIGUES; NAVE, 1999; MIKICH; SILVA, 2001).

Quando analisada cada uma das áreas, as espécies mais abundantes, com suas respectivas síndromes de dispersão e número de indivíduos, foram no PNI: Euterpe edulis (zoocórica; 159 indivíduos); Sorocea bonplandii (zoocórica; 99) e Guarea kunthiana (zoocórica; 57), no FS: Machaerium stipitatum (anemocórica; 37); Chrysophyllum gonocarpum (zoocórica; 26) e Sorocea bonplandii (zoocórica; 23), no FT: Euterpe edulis (zoocórica; 125); Chrysophyllum gonocarpum (zoocórica; 34) e Sorocea bonplandii (zoocórica; 30), no FRi: Inga striata (zoocórica; 68); Handroanthus impetiginosus (anemocórica; 55) e Senegalia polyphylla (autocórica; 43) e no FRt foram: Psidium guajava (zoocórica; 129), Hovenia dulcis (zoocórica; 57) e Machaerium stipitatum (anemocórica; 61). Dentre essas espécies mais abundantes, observase que, para PNI e FT todas apresentam dispersão zoocórica, enquanto para FS e FRt, duas apresentaram dispersão zoocórica e uma anemocórica, e para FRi foram observados os três grupos (uma zoocórica, uma autocórica e uma anemocórica). Esses dados corroboram com Mikich e Silva (2001) para uma área de FES no Paraná, além disso, uma predominância de espécies zoocóricas é esperada para florestas em estágio mais avançados, nas quais a complexidade vegetal aumenta, contribuindo nas interações entre frugívorosplantas, promovendo estabilidade no ecossistema e permitindo a dispersão de diásporos (FENNER, 1985; SILVA, 2008). Toscan et al. (2014) em um estudo sobre chuva de sementes realizado nesse mesmo corredor, amostrando as áreas FT e FRt, comprovaram esse padrão, sendo que a área FT apresentou 57\% de espécies zoocóricas enquanto FRt apresentou $47 \%$ das espécies como anemocóricas.

Observa-se também que, entre os remanescentes florestais nativos (PNI, FT e FS), espécies mais abundantes como Euterpe edulis, Chrysophyllum gonocarpum e Sorocea bonplandii ocorrem em mais de um fragmento, enquanto nas áreas em que houve reflorestamento as maiores abundâncias pertencem a espécies distintas, incluindo exóticas, especialmente na área de FRt.

Das 134 espécies amostradas 33 foram encontradas apenas nas áreas com reflorestamentos (FRt e FRi) e possivelmente não ocorram de forma natural na região estudada, além disso 12 são exóticas (Tabela 1). Desta forma, excetuando Citrus X aurantium, que é amplamente difundido na região, as demais espécies exóticas encontram-se distribuídas apenas nas áreas reflorestadas: FRi (Casuarina equisetifolia), e FRt (Mangifera indica, Leucaena leucocephala, Persea americana, Eucalyptus polyanthemos, Psidium guajava, Ligustrum japonicum, Hovenia dulcis, Eriobotrya japonica, Citrus deliciosa e Citrus limonia), com destaque para Psidium guajava e Hovenia dulcis que estão entre as mais frequentes nessa área, com 129 e 57 indivíduos, respectivamente. Deve ser considerado ainda que sete destas 12 espécies exóticas já foram relatadas como invasoras (INSTITUTO HÓRUS DE DESENVOLVIMENTO E CONSERVAÇÃO AMBIENTAL, 2012).

A abundância de algumas destas espécies exóticas aponta a necessidade de manejo, principalmente das que possuem potencial invasor (como Mangifera indica, Casuarina equisetifolia, Leucaena leucocephala, Psidium guajava e Hovenia dulcis) para que a contaminação biológica das áreas preservadas do CBSM seja evitada (ZILLER, 2001).

As cinco áreas estudadas apresentaram densidade, diversidade e equabilidade bastante distintas (Tabela 2). Em relação à densidade de indivíduos, observa-se que as áreas PNI e FT apresentam valores próximos, enquanto FS demonstrou a mais baixa densidade dentre os remanescentes florestais do Corredor. Em outros trabalhos realizados em Floresta Estacional Semidecidual (IVANAUSKAS; RODRIGUES; NAVE, 1999; CAMPOS; ROMAGNOLO; SOUZA, 2000; BIANCHINI et al., 2003; COSTA-FILHO; NANNI; CAMPOS, 2006; DEL QUIQUI et al., 2007) os valores variaram entre 1239 e 2271 ind./ha (Tabela 2).

As áreas FRi e FRt, apresentaram densidade baixas em relação às áreas de remanescentes (PNI, FT e FS), porém, quando comparadas com outro trabalho realizado em uma área de reflorestamento com espécies nativas de FES (COLMANETTI; BARBOSA, 2013), esse valor está próximo ao esperado. Ambas, 
FRi e FRt, também apresentaram diversidade e equabilidade baixas, mesmo quando comparados a esse estudo também em área de reflorestamento. Isso se deve à dominância de Handroanthus impetiginosus, Inga striata, Senegalia polyphylla e Guazuma ulmifolia que representaram cerca de $56 \%$ de todos os indivíduos no FRi e Psidium guajava e Hovenia dulcis que chegaram a $32 \%$ dos indivíduos amostrados na FRt, enquanto no estudo de Colmanetti e Barbosa (2013) utilizou-se um plantio com alta riqueza, 100 espécies.

O PNI também apresentou baixo valor de diversidade, provavelmente devido à dominância de algumas espécies na área estudada como Euterpe edulis, Guarea kunthiana e Sorocea bonplandii que representaram mais de $61 \%$ dos indivíduos encontrados, quando comparados com outras áreas de mesma formação vegetacional (entre 3,20 e 3,77) (Tabela 2).

O mesmo ocorre para a equabilidade em relação ao PNI, já que os valores encontrados por outros autores em remanescentes de FES variaram entre 0,77 e 0,82 . Apenas os valores de diversidade alcançados para FS e FT encontram-se dentro da faixa obtida para outros remanescentes de mesma formação vegetacional (IVANAUSKAS; RODRIGUES; NAVE, 1999; CAMPOS; ROMAGNOLO; SOUZA, 2000; BIANCHINI et al., 2003; COSTA-FILHO; NANNI; CAMPOS, 2006; DEL QUIQUI et al., 2007).

\section{Similaridade florística}

Os dendrogramas que representam a similaridade florística (índices de Bray Curtis e Jaccard) entre as áreas de estudo no CBSM (Figura 2) evidenciam uma maior similaridade entre o PNI e o FT, seguida da área FS. Além disso, a distribuição do número de indivíduos entre as espécies assemelha-se bastante entre as três áreas, principalmente entre o FT e o PNI, como pode ser constatado pela abundância das espécies Guarea kunthiana e Euterpe edulis (Tabela 1), que são dominantes apenas nessas duas áreas. Já as áreas FRi e FRt, se apresentaram muito diferentes do PNI, uma vez que foram reflorestadas com espécies de baixa

TABELA 2: Área amostral em hectares (Área), circunferência a altura do peito (CAP), densidade (D) em indivíduos por hectare, diversidade de Shannon-Wiener (H') e equabilidade de Pielou (J') para as cinco áreas amostradas no Corredor de Biodiversidade Santa Maria (PR) e para outros trabalhos realizados em remanescentes de FES.

TABLE 2: Sample area in hectares (Area), circumference at breast height (CAP), density (D) of individuals per hectare, Shannon-Wiener (H') and evenness (J') for the five sampled areas in Santa Maria Biodiversity Corridor, PR state, and for the other studies conducted in remnants of FES.

\begin{tabular}{lccccc}
\hline \multicolumn{1}{c}{ Local } & \multicolumn{1}{c}{ Presente trabalho } & & & \\
\hline & Área & CAP & D & H' & J' \\
\hline FS - Fragmento Secundário & 0,40 & 15 & 915 & 3,66 & 0,87 \\
FT - RPPN Fazenda Santa Maria & 0,40 & 15 & 1285 & 3,37 & 0,79 \\
FRi - Fragmento Reflorestado Inicial & 0,40 & 15 & 885 & 2,65 & 0,81 \\
FRt - Fragmento Reflorestado Tardio & 0,40 & 15 & 1440 & 3,00 & 0,75 \\
PNI - Parque Nacional do Iguaçu & 0,40 & 15 & 1295 & 2,71 & 0,67 \\
\hline \multicolumn{1}{c}{ Outros trabalhos realizados em FES } & & & & \\
\hline \multicolumn{1}{c}{ Local/Referência } & Área & CAP & D & H' & J' \\
\hline Parque Estadual Mata dos Godoy- PR (BIANCHINI et al., 2003) & 0,50 & 15 & 1824 & 3,44 & - \\
Estação Ecológica do Caiuá - PR (COSTA-FILHO et al., 2006) & 2,25 & - & 1239 & 3,32 & 0,77 \\
Estação Ecológica do Caiuá - PR (DEL QUIQUI et al., 2007) & 4,35 & 15,7 & - & 3,56 & - \\
Alto Rio Paraná - PR (CAMPOS; ROMAGNOLO; SOUZA, 2000) & 0,54 & 15 & 1472 & 3,20 & - \\
Fazenda Santa Irene - SP (IVANAUSKAS et al., 1999) & 0,42 & 15 & 2271 & 3,77 & 0,82 \\
RPPN Parque Florestal São Marcelo - SP (COLMANETTI; BARBOSA, 2013) & 0,45 & 15 & 809 & 3,87 & 0,89 \\
\hline
\end{tabular}


TABELA 3: Valores do teste de Hutcheson (T) em nível de $5 \%$ de significância nas comparações par a par dos índices de diversidade das cinco áreas amostradas no Corredor de Biodiversidade Santa Maria (PR).

TABLE 3: Hutcheson test (T) at 5\% significance level for diversity index in the five sampled areas of Santa Maria Biodiversity Corridor.

\begin{tabular}{lcc}
\hline & $\mathrm{p}$ & $\mathrm{T}$ \\
\hline PNI-FS & $<0,05$ & 10,3890 \\
PNI-FT & $<0,05$ & 6,8445 \\
PNI-Fri & $>0,05$ & $-0,40904$ \\
PNI-FRt & $<0,05$ & 3,3448 \\
FS-FT & $<0,05$ & $-3,3579$ \\
FS-Fri & $<0,05$ & $-12,8750$ \\
FS-FRt & $<0,05$ & $-8,1396$ \\
FT-Fri & $<0,05$ & $-8,4048$ \\
FT-FRt & $<0,05$ & $-4,1722$ \\
FRt-Fri & $<0,05$ & 4,4391 \\
\hline
\end{tabular}

ocorrência, ou até mesmo ausentes, nos demais fragmentos estudados.

PNI e FT compartilham um total de 43 espécies, sendo que quatro (Aspidosperma polyneuron, Euterpe edulis, Aegiphilla integrifolia e Eugenia burkartiana) ocorreram exclusivamente nessas áreas.

A espécie Euterpe edulis, o palmito, também apresentou as maiores abundâncias nestas duas áreas (Tabela 1). Esta espécie tende a ocorrer em grande densidade de forma natural (FAVRETO, 2010), sendo uma espécie clímax (CARPANEZZI; CARPANEZZI, 2006). Assim, as áreas em que ela ocorre no CBSM se encontram em avançado estádio sucessional. Outra espécie clímax que ocorreu na PNI e na FT foi
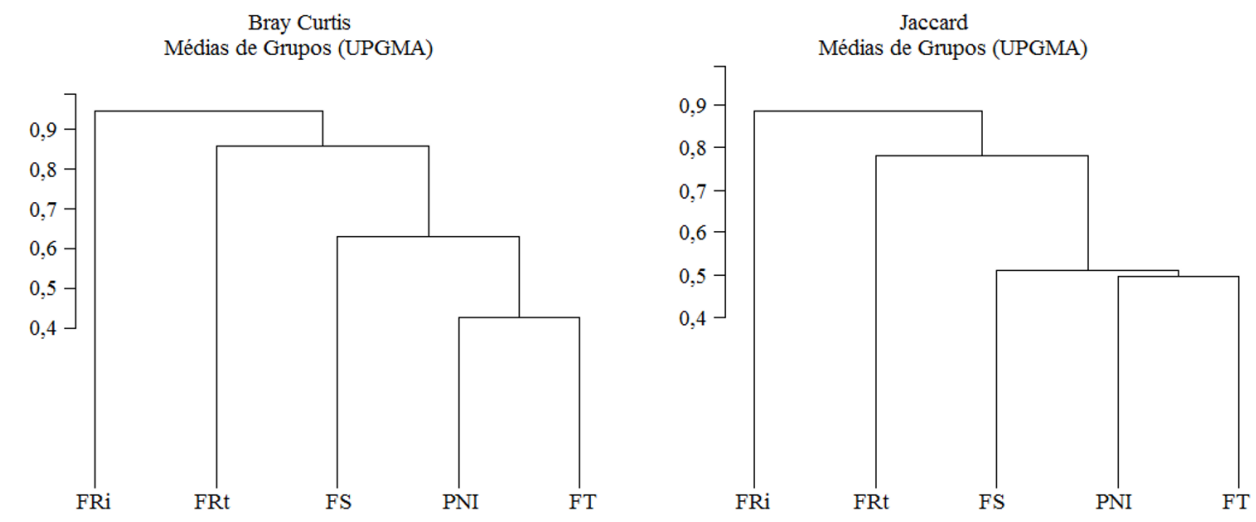

FIGURA2: Dendrogramas de similaridade florística a partir dos dados de densidade (índice de dissimilaridade de Bray Curtis) e presença/ausência (índice de similaridade de Jaccard) das espécies para as cinco áreas analisadas no Corredor de Biodiversidade Santa Maria. Parque Nacional do Iguaçu (PNI): Fragmento Tardio (FT), Fragmento Secundário (FS), Fragmento Reflorestado Inicial (FRi) e Fragmento Reflorestado Tardio (FRt).

FIGURE 2: Dendrograms of floristic similarity from density data (Bray Curtis distance) and the presence/ absence of data (Jaccard similarity index) of the species for the five sampled areas analyzed for Santa Maria Biodiversity Corridor. Iguaçu National Park (PNI): Late Fragment (FT), Secondary Fragment (FS), Initial Reforested Fragment (FRi) and Late Reforested Fragment (FRt). 
Aspidosperma polyneuron, a peroba-rosa, árvore de ocorrência natural rara, que se encontra ameaçada de extinção (INTERNATIONAL UNION FOR CONSERVATIONS OF NATURE, 2015), por possuir madeira de ótima qualidade muito procurada pela indústria moveleira.

Apesar do FS se situar geograficamente mais próximo do PNI, compartilhou um número maior de espécies (45) com FT, das quais sete não ocorrem no PNI. Provavelmente, tal semelhança se deve ao fato dos fragmentos terem um histórico de antropização, serem muito menores que PNI e possuírem uma mata em regeneração, na qual os efeitos de borda e clareira são muito mais frequentes.

Por outro lado, o FRi e o FRt foram consideradas áreas muito diferentes floristicamente do PNI (Figura 2) provavelmente devido ao fato destas áreas terem sido reflorestadas com a utilização de arranjos com baixa riqueza de espécies, sendo muitas delas exóticas.

Diversos fatores abióticos afetam diretamente a distribuição e a dinâmica das populações vegetais, como a ocorrência de distúrbios (NUNES et al., 2003), o grau de preservação da área, a proximidade de corpos d'água, tipo e qualidade do solo e pluviometria. Desta forma, a maior similaridade entre os remanescentes florestais (PNI, FT e FS) podem estar relacionadas a esses fatores, bem como ao histórico e grau de antropização de cada uma das áreas, já a dissimilaridade com as áreas reflorestadas (FRt e FRi), se deve, além desses fatores, ao tipo de manejo que foi empregado no processo de recuperação de cada uma delas.

\section{CONCLUSÕES}

As diferentes áreas do Corredor de Biodiversidade Santa Maria apresentam densidade, diversidade e composições bastante distintas, principalmente entre as áreas reflorestadas e as florestas nativas preservadas em diferentes estágios médio e avançado de sucessão.

Houve dominância de algumas espécies como Euterpe edulis nas áreas mais preservadas, diminuindo assim, os valores de diversidade, porém, observa-se também que, nessas áreas mais preservadas, as espécies dominantes são de dispersão zoocórica, sendo essa predominância de zoocoria um padrão comum em áreas em estágios avançados.

A dissimilaridade com as áreas nativas e a abundância de indivíduos de espécies exóticas nas áreas reflorestadas demonstram a baixa efetividade dessas em desenvolver a flora nativa. Vale ressaltar que as espécies exóticas encontradas nessas áreas reflorestadas ainda não foram encontradas nas áreas mais preservadas e, portanto, o manejo das mesmas deve ser incentivado, evitando sua disseminação, como medida de preservação dos fragmentos florestais remanescentes.

\section{AGRADECIMENTOS}

À equipe do Museu Botânico Municipal de Curitiba pelo auxílio nas identificações e Adaíldo Policena pelo auxílio em campo. Ao Parque Tecnológico de Itaipu, PTI C\&T/FPTI-BR, pela concessão de bolsa de mestrado concedida à primeira autora, à Fundação Araucária (edital 14/2009/ convênio 223/2010), FAPESP - Fundação de Amparo à Pesquisa do Estado de São Paulo (processo/grant no 2010/17400-3), CNPq - Conselho Nacional de Desenvolvimento Científico e Tecnológico (processo/grant n ${ }^{\circ}$ 562240/20101) pelos auxílios financeiros.

\section{REFERÊNCIAS}

ANGIOSPERM PHYLOGENY GROUP. An update of the Angiosperm Phylogeny Group classification for the orders and families of flowering plants: APG III. Botanical Journal of the Linnean Society, Londres, v. 161, p. 105-121, 2009.

BHERING, S. B.; SANTOS, H. G. Mapa de Solos do Estado do Paraná: legenda Atualizada. Rio de Janeiro: Embrapa Florestas; Embrapa Solos; IAPAR, 2008. 234 p.

BIANCHINI, E. et al. Diversidade e estrutura de espécies arbóreas em área alagável do município de Londrina, Sul do Brasil. Acta Botanica Brasilica, São Paulo, v. 17, n. 3, p. 405-419, 2003.

BRIDSON, D.; FORMAN, L. The herbarium handbook. Kew: The Royal Botanic Garden, 2004. 334 p. 
CAMPOS, J. B.; ROMAGNOLO, M. B.; SOUZA, M. C. Structure, composition and spatial distribution of tree species in a remnant of the semideciduous seasonal alluvial forest of the upper Paraná River Floodplain. Brazilian Archives of Biology and Technology, Curitiba, v. 43, n. 2, p. 185-194, 2000.

CAMPOS, J. B. A fragmentação de ecossistemas, efeitos decorrentes e corredores de biodiversidade. In: CAMPOS, J. B.; TOSSULINO, M. G. P.; MULLER, C. R. C. (Org.). Unidades de conservação: ações para a valorização da biodiversidade. Curitiba: Instituto Ambiental do Paraná, 2006. p. 165-174.

CARPANEZZI, A. A.; CARPANEZZI, O. T. B. Espécies nativas recomendadas para recuperação ambiental no Estado do Paraná, em solos não degradados. Colombo: Embrapa Florestas, 2006. 57 p.

COLMANETTI, M. A. A.; BARBOSA, L. M. Fitossociologia e estrutura do estrato arbóreo de um reflorestamento com espécies nativas em Mogi-Guaçu, SP, Brasil. Hoehnea, São Paulo, v. 40, n. 3, p. 419-435, 2013.

COSTA-FILHO, L. V.; NANNI, M. R.; CAMPOS, J. B. Floristic and Phytosociological Description of a Riparian Forest and the Relationship with the Edaphic Environment in Caiuá Ecological Station - Paraná Brazil. Brazilian Archives of Biology and Technology, Curitiba, v. 49, n. 5, p. 785-798, 2006.

DEL QUIQUI, E. M. et al. Estudo fitossociológico de um trecho da Floresta Estacional Semidecidual em Diamante do Norte, Estado do Paraná, Brasil. Acta Scientiarum Agronomy, Maringá, v. 29, n. 2 , p. 283-290, 2007.

FAVRETO, R. Aspectos etnoecológicos e ecofisiológicos de Euterpe edulisMart. (Arecaceae). 2010. 143 f. Tese (Doutorado em Botânica) - Universidade Federal do Rio Grande do Sul, Porto Alegre, 2010.

FENNER, M. Seed ecology. London: Chapman and Hall, 1985. 151 p.

HANSKI, I.; GILPIN, M. Metapopulation dynamics: brief history and conceptual domain. Biological Journal of Linnean Society, Londres, v. 42, p. 3-16, 1991.

HUTCHESON, K. A Test for Comparing Diversities based on the Shannon Formula. Journal of the oretical Biology, Londres, v. 29, p. 151-154, 1970.

IBAMA. Corredor de Santa Maria. 2012. Disponível em: <http://www.prbiodiversidade.pr.gov.br/ arquivos/File/36\%20IBAMACORREDOR_SANTA_MARIA.pdf.>. Acesso em: 10 jan. 2012.

INTERNATIONAL UNION FOR CONSERVATIONS OF NATURE. Americas Regional Workshop (Conservation \& Sustainable Management of Trees, Costa Rica, November 1996) 1998. Aspidospermapolyneuron. The IUCN Red List of Threatened Species. Version 2015.2. 2015. Disponível em: <http://www.iucnredlist.org/details/32023/0>. Acesso em: 29 jul. 2015.

INSTITUTO HÓRUS DE DESENVOLVIMENTO E CONSERVAÇÃO AMBIENTAL . [2012]. Análise de risco para espécies exóticas. Disponível em: $<$ http://www.institutohorus.org.br/index.php?modulo=inf_ analise_risco>. Acesso em: 16 jan. 2012.

ITAIPU BINACIONAL. Corredor de Biodiversidade. 2010. Disponível em: <http://www.itaipu.gov.br/ index.php?q=node/239>. Acesso em: 14 ago. 2010.

IVANAUSKAS, N. M.; RODRIGUES, R. R.; NAVE, A. G. Fitossociologia de um trecho de Floresta Estacional Semidecidual em Itatinga, São Paulo, Brasil. Scientia Forestalis, Piracicaba, v. 56, p. 83-99, 1999.

JARDIM BOTÂNICO DO RIO DE JANEIRO. Lista de espécies da flora do Brasil. 2013. Disponível em: $<$ http://floradobrasil.jbrj.gov.br.>. Acesso em: 03 abr. 2013.

LORENZI, H. Árvores Brasileiras: manual de identificação e cultivo de plantas arbóreas do Brasil.

São Paulo: Nova Odessa, 2002a. v. 1.

LORENZI, H. Árvores Brasileiras: manual de identificação e cultivo de plantas arbóreas do Brasil. São Paulo: Nova Odessa, 2002b. v. 2.

LORENZI, H. Árvores Brasileiras: manual de identificação e cultivo de plantas arbóreas do Brasil. São Paulo: Nova Odessa, 2010. v. 3.

MAACK, R. Geografia física do estado do Paraná. 4. ed. Ponta Grossa: Editora UEPG, 2012. 526 p.

METZGER, J. P.; DÉCAMPS, H. The structural connectivity threshold: an hypothesis in conservation biology at the landscape scale. Acta Oecologica, Fribourg, v. 18, n. 1, p. 1-12, 1997.

MIKICH, S. B.; SILVA, S. M. Composição florística e fenologia das espécies zoocóricas de remanescentes de Floresta Estacional Semidecidual no Centro-Oeste do Paraná, Brasil. Acta Botanica Brasilica, São Paulo, v. 15, n. 1, p. 89-113, 2001. 
MUELLER-DOMBOIS, D.; ELLENBERG, H. Aims and methods of vegetation ecology. New York: John Wiley \& Sons, 1974. 574 p.

MYERS, N. et al. Biodiversity hotspots for conservation priorities. Nature, Londres, v. 403, p. 853-858, 2000.

NUNES, Y. R. F. et al. Variações da fisionomia, diversidade e composição de guildas da comunidade arbórea em um fragmento de Floresta Semidecidual em Lavras, MG. Acta Botanica Brasilica, São Paulo, v. 17, n. 2, p. 213-229, 2003.

PINTO, L. P. et al. Mata Atlântica brasileira: os desafios para a conservação da biodiversidade de um hotspot mundial. In: ROCHA, C. F. D. et al. (Org.). Biologia da conservação: essências. São Carlos: RiMa, 2006. p. 91-118.

POLA, I. R. V. Explorando conceitos da teoria de espaços métricos em consultas por similaridade sobre dados complexos. 2010. 169 f. Tese (Doutorado em Ciência da Computação e Matemática Computacional) - Universidade de São Paulo, São Carlos, 2010.

R CORE TEAM. R: a language and environment for statistical computing. R Foundation for Statistical Computing, Vienna: R Foundation for Statistical Computing, 2013. Disponível em : <http://www.R-project. org/>. Acesso em : 08 nov. 2015.

RAMOS, V. S. et al. Árvores da Floresta Estacional Semidecidual: guia de identificação de espécies. São Paulo: Edusp-Fapesp, 2008. 320 p.

ROCHA, C. F. D. et al. Corredores Ecológicos e Conservação da Biodiversidade: um Estudo de Caso na Mata Atlântica. In: ROCHA, C. F. D. et al. (Org.). Biologia da conservação: essências. São Carlos: RiMa, 2006. p. 293-318.

RODRIGUES, R. R.; GANDOLFI, S. Conceitos, Tendências e Ações para a Recuperação de Florestas Ciliares. In: RODRIGUES, R. R.; LEITÃO-FILHO, H. F. (Org.). Matas Ciliares: conservação e recuperação. São Paulo: Edusp; Fapesp, 2004. p. 235-247.

SALAMUNI, R. et al. Parque Nacional do Iguaçu, PR - Cataratas de fama mundial. In: SCHOBBENHAUS, C. et al. (Org.). Sítios Geológicos e Paleontológicos do Brasil. Brasília: DNPM/CPRM/SIGEP, 2002. p. 313-312.

SANTOS, L. J. C. et al. Mapeamento geomorfológico do Estado do Paraná. Revista Brasileira de Geomorfologia, São Paulo, v. 7, p. 3-11, 2006.

SHEPERD, G. J. FITOPAC 2.1.2: manual do usuário. Campinas: UNICAMP, 2011. 93 p.

SISTEMA METEOROLÓGICO DO PARANÁ. Dados de precipitação e temperatura diários para a cidade de Foz do Iguaçu fornecidos sob consulta no período de 01/01/2010 a 01/01/2011. [2011]. Disponível em: <http://www.simepar.br/>. Acesso em: 10 jan. 2012.

SILVA, W. R. A importância das interações planta-animal nos processos de restauração. In: KAGEYAMA, P. Y. et al. (Org.). Restauração Ecológica de Ecossistemas Naturais. 1. ed. Botucatu: Fepaf, 2008. p. 77-90.

TOSCAN, M. A. G. et al. Litter production and seed rain in semideciduous forest fragments at diferente successional stages in the western part of the state of Paraná. Acta Botanica Brasilica, São Paulo, v. 28, n. 3, p. 392-403, 2014.

VANDER PIJL, L. Principles of Dispersal in Higher Plants. 3. ed. New York: Springer Verlag, 1982. $156 \mathrm{p}$.

VELOSO P. H. P.; FILHO, A. L. R. R.; LIMA, J. C. A. Classificação da Vegetação Brasileira, Adaptada a um Sistema Universal. Rio de Janeiro: IBGE, 1991. 123 p.

ZILLER, S. R. Plantas exóticas invasoras: a ameaça da contaminação biológica. Ciência Hoje, Rio de Janeiro, v. 30, n. 178, p. 77-79, 2001. 